\title{
Dexmedetomidine ameliorates lipopolysaccharide-induced acute lung injury by inhibiting the PI3K/Akt/FoxO1 signaling pathway
}

\author{
Haibin Cui ${ }^{1} \cdot$ Qian Zhang ${ }^{1}$
}

Received: 10 June 2020 / Accepted: 13 February 2021 / Published online: 5 April 2021

C Japanese Society of Anesthesiologists 2021

\begin{abstract}
Purpose Dexmedetomidine (DEX) has been associated with inflammation, oxidative stress, and apoptosis, but its effects on lipopolysaccharide (LPS)-induced lung injury remain uncertain. The present study explored the effects of DEX on LPSinduced lung injury and studied the possible molecular mechanisms by testing the effects of the phosphoinositide-3 kinase (PI3K) inhibitor LY294002 and BEZ235.

Methods Seventy C57BL/6 mice were randomly divided into the control, LPS, LPS + DEX, LPS + LY294002, LPS + BEZ235, LPS + DEX + LY294002, and LPS + DEX + BEZ235groups. Lung samples were collected 48 h after LPS treatment.

Results DEX significantly inhibited LPS-induced increases in the lung weight/body weight ratio and lung wet/dry weight ratio, decreased inflammatory cell infiltration, and decreased the production of proinflammatory factors, such as interleukin- $1 \beta$ (IL-1 $\beta$ ), IL-6, and tumor necrosis factor $\alpha(\mathrm{TNF}-\alpha)$ in the lungs. DEX also markedly attenuated the increases in malondialdehyde 5 (MDA 5) and inositol-dependent enzyme a (IRE-a), attenuated the decrease in superoxide dismutase 1(SOD-1), reversed the low expression of B-cell lymphoma-2 (Bcl-2), and the high expressions of Bax and Caspase-3. DEX also decreased the expression of phosphorylated PI3K and phosphorylated Akt and increased the expression of phosphorylated forkhead box-O transcription factor 1 (FoxO1). More interestingly, LY294002 or BEZ235 pretreatment significantly abolished the inhibitory effects of DEX on LPS-induced lung inflammation, oxidative stress, and apoptosis.

Conclusions These data suggest that DEX ameliorates LPS-induced acute lung injury partly through the PI3K/Akt/FoxO1 signaling pathway.
\end{abstract}

Keywords Dexmedetomidine $\cdot$ Lipopolysaccharide $\cdot$ Acute lung injury $\cdot$ Oxidative stress $\cdot$ Inflammation $\cdot$ PI3K/Akt/FoxO1 signaling pathway

\section{Introduction}

Acute lung injury is a serious clinical complication that is associated with high mortality and morbidity rates of approximately $30 \%$ to $40 \%$. Acute lung injury is usually characterized by the aggregation of inflammatory cells, an increase in vascular and epithelial permeability, interstitial

Qian Zhang

zhangqian_201801@163.com

Haibin Cui

cuihaibin1980@sina.com

1 Department of Anesthesiology, School and Hospital of Stomatological, China Medical University, Liaoning Provincial Key Laboratory of Ora Disease, No. 117 Nanjing North Street, Heping District, Shenyang 110002, China edema, gas exchange abnormalities, and alveolar septal injury [1]. Unfortunately, there are few effective drugs to treat acute lung injury. Lipopolysaccharide (LPS) is a component of Gram-negative bacteria that usually leads to an inflammatory response and lung injury. Lipopolysaccharideinduced acute lung injury is a widely accepted animal model of bacterial sepsis. In vivo experiments showed that intranasal LPS administration dramatically increased inflammatory cell infiltration, oxidative stress, and apoptosis [2,3].

Phosphatidylinositol-3-kinase (PI3K) is an intracellular phosphatidylinositol kinase, and Akt is the direct downstream effector of PI3K. Forkhead box-O transcription factor 1 (FoxO1) is a downstream protein target of Akt. Once Akt is activated, phosphorylated FoxO1 (p-FoxO1) is dephosphorylated to form FoxO1, which is then transferred to the nucleus. The PI3K/Akt/FoxO1 signaling pathway has been 
implicated in type 2 diabetes [4], breast cancer [5], kidney injury [6], and ischemia/reperfusion injury [7, 8]. The PI3K inhibitor LY294002 and the knockdown of PI3K by small interfering RNA dramatically inhibited Akt phosphorylation, reduced the production of human heme oxygenase 1 (HO-1), and then abrogated the effects of hemin pretreatment on LPS-induced lung injury in rats, suggesting that the PI3K/Akt pathway protects lung tissue against oxidative injury in the setting of sepsis [9]. Additionally, a previous study reported that pretreatment with schisandrin alleviated LPS-induced endothelium and epithelium injury of the lungs, which was attributed to the inhibition of cell injury and activation of cell regeneration via regulation of the Akt/ FoxO1 signaling pathway [10]. These studies suggest that $\mathrm{Akt} / \mathrm{FoxO} 1$ may be a key pathway that is involved in LPSinduced acute lung injury. Therefore, targeting this pathway may alleviate LPS-induced acute lung injury and associated complications.

Dexmedetomidine (DEX), a highly selective agonist of the $\alpha 2$-adrenergic receptor, is clinically used for the sedation of ill patients. Clinical indications of DEX: it is used for sedation during tracheal intubation and mechanical ventilation in patients undergoing general anesthesia; and it is also used for sedation of patients who start intubation and use ventilator during intensive care. Continuous infusion of DEX should not exceed $24 \mathrm{~h}$. Clinically, the adult dose is $4 \mu \mathrm{g} / \mathrm{kg}$, drip slowly for $10 \mathrm{~min}$, and then maintain infusion at $0.2-0.7 \mu \mathrm{g} / \mathrm{kg} / \mathrm{h}$ [11]. DEX exerts sedative and analgesic effects and has been reported to protect against LPS-induced acute lung injury through antiinflammatory and immuneregulatory actions $[12,13]$. Several studies reported that pretreatment with DEX ameliorated LPS-induced acute lung injury [14-16]. Moreover, DEX was reported to alleviate sepsis-induced acute lung injury partly through suppression of the production of proinflammatory cytokines that are regulated by the Toll-like receptor 4 (TLR-4)/nuclear factor $\kappa B(N F-k B)$ signaling pathway [17]. However, unknown are whether DEX ameliorates LPS-induced lung inflammation, oxidative stress, and apoptosis and whether the protective effect of DEX involves the PI3K/Akt/FoxO1 signaling pathway. The present study investigated the effects of DEX on LPS-induced lung injury and explored the possible mechanisms of action.

\section{Methods}

\section{Animals and experimental groups}

Seventy male C57BL/6 mice (6-8 weeks old, $20-25 \mathrm{~g}$ ) were obtained from the Experimental Animal Department of China Medical University. All of the mice were kept in cages under standard laboratory conditions at a stable temperature $\left(20-24{ }^{\circ} \mathrm{C}\right)$ and humidity $(55 \% \pm 5 \%)$ with a $12 \mathrm{~h} / 12 \mathrm{~h}$ light/dark cycle. Food and water were provided ad libitum. Seventy mice were randomly divided into seven groups ( $n=10 /$ group): control, LPS, LPS + DEX, LPS + LY294002, LPS + BEZ235, LPS + DEX + LY294002, and LPS + DEX + BEZ235 groups. The intratracheal administration of $10 \mu \mathrm{g}$ LPS (dissolved in $50 \mu \mathrm{l}$ phosphate-buffered saline [PBS]) was to induced acute lung injury [18]. The LPS + DEX and LPS + DEX + LY294002 groups were intraperitoneally injected with $50 \mu \mathrm{g} / \mathrm{kg}$ DEX $0.5 \mathrm{~h}$ before LPS-induced acute lung injury [13]. LY294002 $(5 \mu \mathrm{g} / \mathrm{g})$ and BEZ23530 $(25 \mu \mathrm{g} / \mathrm{g}) 30 \mathrm{~min}$ were intraperitoneally injected before exposed to LPS, respectively, [19, 20], and the control group was exposed only to normal room air. All of the animal experiments were performed in accordance with the Guide for the Care and Use of Laboratory Animals of the National Institutes of Health and Academy of China Medical University.

\section{Sample collection and processing}

Forty-eight hours after LPS treatment, the mice were anesthetized by the intraperitoneal injection of $2 \%$ sodium pentobarbital $(50 \mathrm{mg} / \mathrm{kg})$. All of the mice were sacrificed to collect lung samples. The left lung was immersed in $10 \%$ formalin buffer for histological analysis, and the upper lobe of the right lung was dried with filter paper and weighed on an analytical balance to determine the wet weight. Afterward, the lung samples were dried in an oven at $60{ }^{\circ} \mathrm{C}$ for $72 \mathrm{~h}$, and the dry weight was recorded. The wet weight/ dry weight (W/D) ratio was calculated to indicate edema formation. The remaining lung tissues were frozen in liquid nitrogen for protein analysis.

\section{Histological analysis}

For the histological analysis, the left lung was fixed in $10 \%$ formaldehyde at room temperature, processed, and embedded in paraffin blocks using a Leica Microsystem tissue processor (ASP 300S, Germany). For histological staining, $4-\mu \mathrm{m}$-thick sections were sliced using a Leica Microsystem microtome (Model RM 2265, Germany), which was stained with hematoxylin and eosin (H\&E).

\section{Reactive oxygen species detection}

Lung tissue was stained with dimethoxy 2,3-1,4-naphthoquinone (1:100; catalog no. D5439; Sigma, St. Louis, MO, USA) for $15 \mathrm{~min}$ and then examined under a fluorescence microscope (Olympus, Tokyo, Japan). 


\section{TUNEL staining}

Terminal deoxynucleotidyl transferase dUTP nick-end labeling (TUNEL) staining was performed according to the TUNEL kit manufacturer's instructions (Abcam, UK). The nuclei of apoptotic cells were stained brown. Five fields were randomly selected under a high-power microscope $(400 \times$ magnification) to calculate the apoptotic index.

\section{Immunofluorescence staining}

Immunofluorescence staining was performed to detected p-PI3K (1:200, Cell Signaling Technology, USA), p-Akt (1:400, Cell Signaling Technology, USA), and p-FoxO1 (1:250, Abcam, UK) using appropriate antibodies. Lung tissues were dewaxed with xylene, dehydrated through a graded series of alcohol, incubated with $0.1 \%$ Triton X-100 for $30 \mathrm{~min}$, and washed three times with PBS for $5 \mathrm{~min}$ each time. Samples were blocked with 5\% bovine serum albumin and $10 \%$ goat serum for 30 min each, incubated with primary antibody overnight at $4{ }^{\circ} \mathrm{C}$ in a wet box, and stained with a fluorescent secondary antibody. Finally, the samples were observed and photographed under a microscope.

\section{Western blot analysis}

Lung tissues were lysed in complete RIPA buffer $(10 \mathrm{mM}$ Tris- $\mathrm{HCl}$ [pH 7.4], $150 \mathrm{mM} \mathrm{NaCl}, 1 \% \mathrm{NP} 40,0.1 \%$ sodium dodecyl sulfate, $1 \mathrm{mM}$ phenylmethylsulfonyl fluoride, and $1 \times$ protease inhibitor cocktail) and homogenized using a
Sonic Dismembrator 100 (Fisher Scientific, Rockford IL, USA). The protein concentration of the tissue homogenates was measured, and equal amounts of soluble protein were separated on $10 \%$ polyacrylamide gels and transferred onto a nitrocellulose membrane, followed by routine Western blot analysis using appropriate primary and secondary antibodies (Table 1). Proteins were visualized using a ClarityTM Western ECL Substrate and a Tanon 5200 Full automatic chemiluminescence image analysis system (Tanon Science and Technology, Shanghai, China).

\section{Statistical analysis}

The statistical analysis was performed using SPSS 26.0 software (IBM, Armonk, NY, USA). The data are expressed as mean \pm standard error of the mean (SEM) and were analyzed using $t$-tests and one-way analysis of variance (ANOVA). Two-tailed values of $p<0.05$ were considered statistically significant.

\section{Results}

\section{Dexmedetomidine ameliorated LPS-induced acute lung injury in mice}

No mouse was dead before $48 \mathrm{~h}$. Compared with the control group, the lung weight/body weight ratio and W/D ratio were significantly higher in the LPS group, and DEX significantly inhibited these LPS-induced increases (Fig. 1a-b,
Table 1 Primary antibody and secondary antibody list

\begin{tabular}{|c|c|c|c|}
\hline Antibody & Dilution & Catalogue no & Manufacturer \\
\hline IL-1 $\beta$ & $1: 2000$ & sc7884 & Santa Cruz Biotechnology, USA \\
\hline TNF- $\alpha$ & $1: 2000$ & ab8348 & Abcam, UK \\
\hline IL-6 & $1: 1000$ & $a b 83053$ & Abcam, UK \\
\hline IL-10 & $1: 2000$ & ab9969 & Abcam, UK \\
\hline MDA5 & $1: 200$ & ab69983 & Abcam, UK \\
\hline IRE $\alpha$ & $1: 2000$ & 3294 & Abcam, UK \\
\hline SOD-1 & $1: 1000$ & sc11407 & Santa Cruz Biotechnology, USA \\
\hline Bax & $1: 2000$ & $\operatorname{sc} 526$ & Santa Cruz Biotechnology, USA \\
\hline Caspase 3 & $1: 500$ & sc7148 & Santa Cruz Biotechnology, USA \\
\hline Bcl-2 & $1: 1000$ & sc7382 & Santa Cruz Biotechnology, USA \\
\hline PI3K & $1: 1000$ & ab186612 & Abcam, UK \\
\hline p-PI3K & $1: 1000$ & $4228 \mathrm{~S}$ & Cell Signaling Technology, USA \\
\hline Akt & $1: 2000$ & $a b 32505$ & Abcam, USA \\
\hline p-Akt & $1: 2000$ & $4051 \mathrm{~S}$ & Cell Signaling Technology, USA \\
\hline FoxO1 & $1: 1000$ & 2880 & Cell Signaling Technology, USA \\
\hline p-FoxO1 & $1: 1000$ & ab131339 & Abcam, UK \\
\hline GAPDH & $1: 5000$ & sc32233 & Santa Cruz Biotechnology, USA \\
\hline $\begin{array}{l}\text { Horseradish peroxidase-conju- } \\
\text { gated goat anti-mouse IgG }\end{array}$ & 1: 4000 & ab6789 & Abcam, UK \\
\hline
\end{tabular}


A

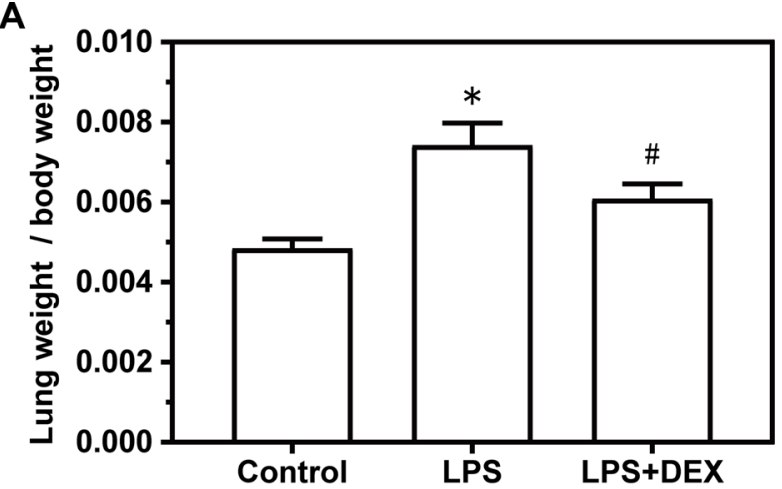

B

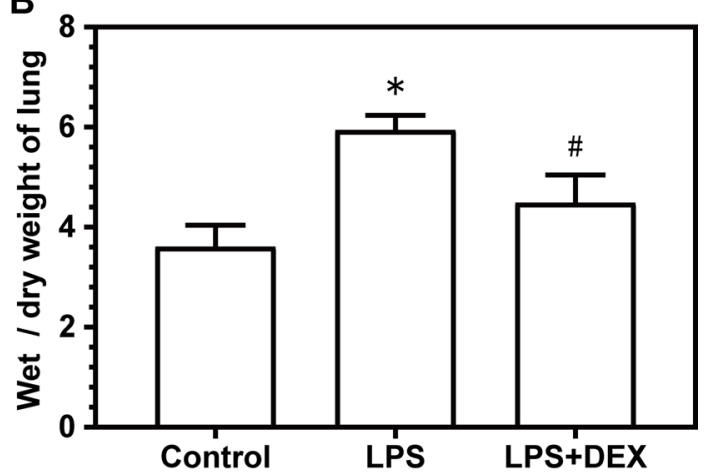

C

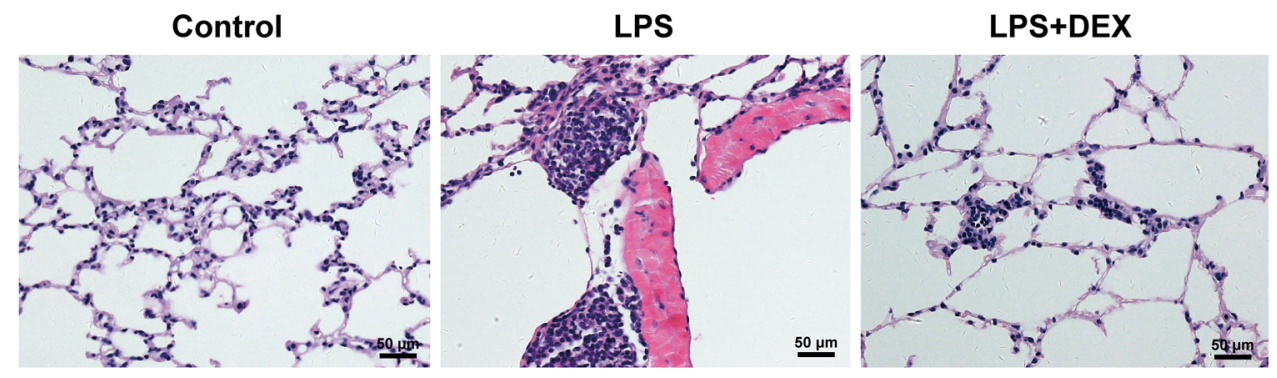

D

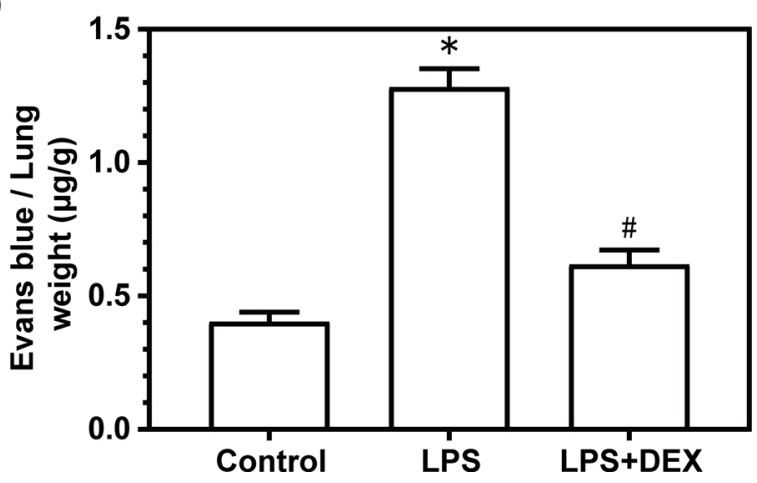

Fig. 1 Dexmedetomidine ameliorated LPS-induced acute lung injury. a, b Lung weight/body weight ratio and wet/dry ratio. c Representative histopathological images of each group (HE staining, scale bar $=50 \mu \mathrm{m})$. d Evans blue staining was used to evaluate changes in vascular permeability. ${ }^{*} p<0.05$, vs. control group; ${ }^{\#} p<0.05$, vs. LPS group $p<0.05)$. Compared with the control group, LPS treatment caused significant lung injury, manifested as hemorrhage, edema, inflammatory cell infiltration, and an increase in Evans blue leakage, and these deleterious effects were significantly ameliorated by DEX treatment (Fig. $1 \mathrm{c}-\mathrm{d}, p<0.05)$.

\section{Dexmedetomidine attenuated the LPS-induced production of proinflammatory factors in the lungs}

It was reported that obviously lung edema, inflammatory cells infiltration and apoptosis were observed in mice treated with LPS for $48 \mathrm{~h}$. Therefore, the protective effect and mechanism of DEX on the lungs of mice treated with LPS were studied. The LPS group exhibited significant increases in interleukin-1 $\beta$ (IL-1 $\beta$ ), IL-6, and tumor necrosis factor
$\alpha(\mathrm{TNF}-\alpha)$ expression and a significant decrease in IL-10 expression compared with the control group. DEX treatment significantly decreased IL- $1 \beta$, IL- 6 , and TNF- $\alpha$ expression and increased IL-10 expression compared with the LPS group (Fig. 2, $p<0.05$ ).

\section{Dexmedetomidine inhibited LPS-induced oxidative stress in the lungs in mice}

Compared with the control group, reactive oxygen species (ROS) levels significantly increased in the LPS group, while DEX decreased ROS expression (Fig. 3a). The LPS group exhibited significant increases in malondialdehyde 5 (MDA 5) and inositol-dependent enzyme a (IRE-a) expression and a significant decrease in superoxide dismutase 


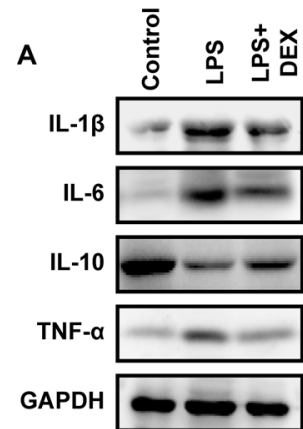

B

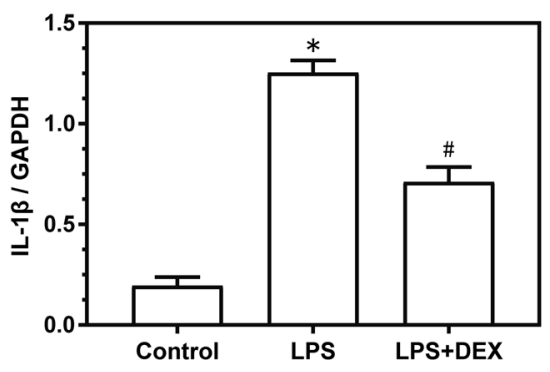

C
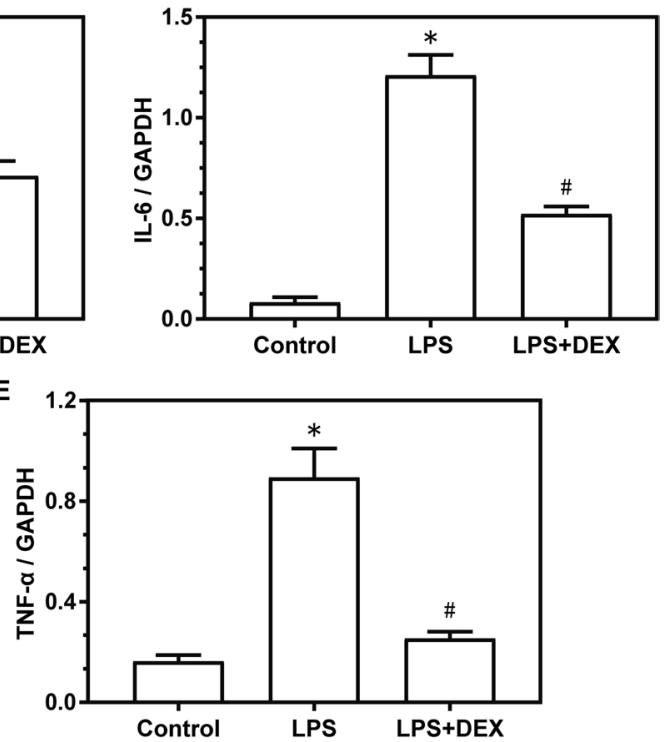

Fig. 2 Dexmedetomidine attenuated LPS-induced inflammation in the lungs. a Representative Western blot images and (b-e) quantitative analysis of IL- $1 \beta$, IL- 6 , IL-10, and TNF- $\alpha$. All of the experi- ments were repeated at least three times. The results are expressed as mean \pm SEM ( $n=10 /$ group). ${ }^{*} p<0.05$, vs. control group; ${ }^{\#} p<0.05$, vs. LPS group
A
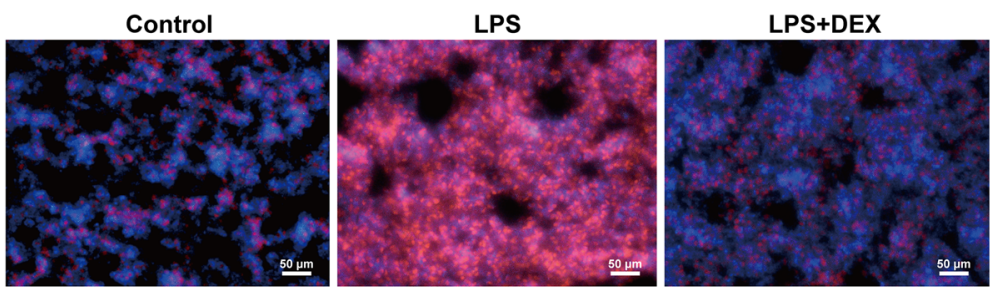

C

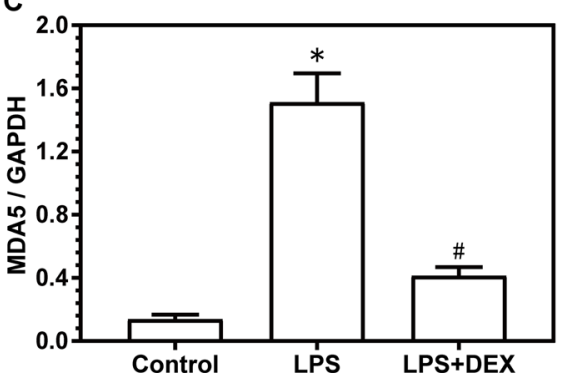

D

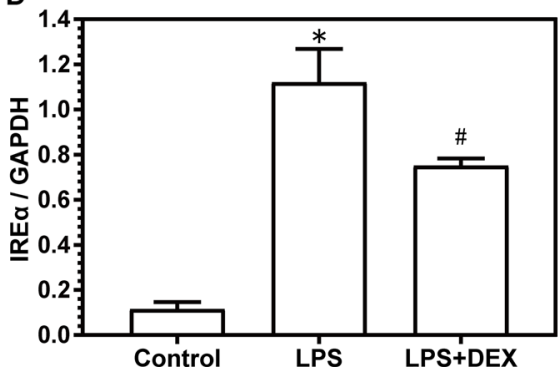

B

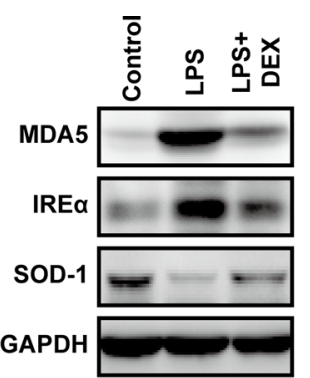

E

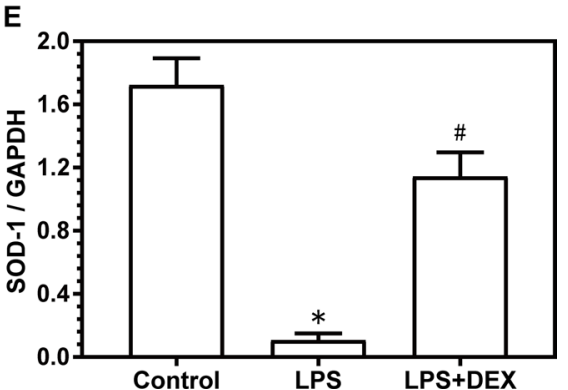

Fig. 3 Dexmedetomidine inhibited LPS-induced oxidative stress in the lungs in mice. a Representative ROS images from lung tissues (scale bar $=50 \mu \mathrm{m}$ ). b-e Representative images and quantitative analysis of MDA5, IRE $\alpha$, and SOD-1.All of the experiments

1 (SOD-1) compared with the control group. DEX treatment significantly alleviated the LPS-induced increases in MDA5 and IRE-a expression and decrease in SOD-1 expression (Fig. 3b-e, $p<0.05$ ). were repeated at least three times. The results are expressed as mean \pm SEM ( $n=10$ /group). ${ }^{*} p<0.05$, vs. control group; ${ }^{\#} p<0.05$, vs. LPS group

\section{Dexmedetomidine decreased LPS-induced apoptosis in lung tissue in mice}

The LPS group exhibited a significant increase in 
TUNEL-positive cells compared with the control group. DEX treatment significantly attenuated the LPS-induced increase in TUNEL-positive cells (Fig. $4 \mathrm{a}-\mathrm{b}, p<0.05$ ). The LPS group also exhibited a significant decrease in Bcl-2 and significant increases in Bax and Caspase- 3 expression compared with the control group. DEX treatment significantly reversed the LPS-induced decrease in Bcl-2 expression and increases in Bax and Caspase-3 expression (Fig. 4c-f, $p<0.05$ ).

\section{Dexmedetomidine reversed the effect of LPS on the PI3K/Akt/Fox01 signaling pathway}

The LPS group exhibited increases in p-PI3K and p-Akt expression and a decrease in p-FoxO1 expression compared with the control group. DEX treatment significantly attenuated the LPS-induced increases in p-PI3K and p-Akt expression and decrease in p-FoxO1 expression (Fig. 5a-d, $p<0.05)$. The LPS group exhibited significant increases in
p-PI3K- and p-Akt-positive cells and a significant decrease in p-FoxO1-positive cells compared with the control group. DEX significantly reversed the increases in p-PI3K- and p-Akt-positive cells and the decrease in p-FoxO1-positive cells (Fig. $5 \mathrm{e}-\mathrm{j}, p<0.05$ ).

\section{LY294002 pretreatment abolished the effects of DEX on LPS-induced acute lung injury}

Compared with the LPS group, LY294002 pretreatment significantly decreased the lung weight/body weight ratio and W/D ratio, decreased the expression levels of p-PI3K, p-Akt, p-FoxO1, IL-1 $\beta$, IL-6, TNF- $\alpha$, MDA5, IRE-a, Bax and Caspase-3, and increased the expression levels of IL-10, SOD-1 and Bcl-2 expression (Fig. 6, $p<0.05$ ). LY294002 pretreatment significantly abolished the inhibitory effects of DEX on LPS-induced lung inflammation, oxidative stress, and apoptosis (Fig. $6, p<0.05$ ). These
A
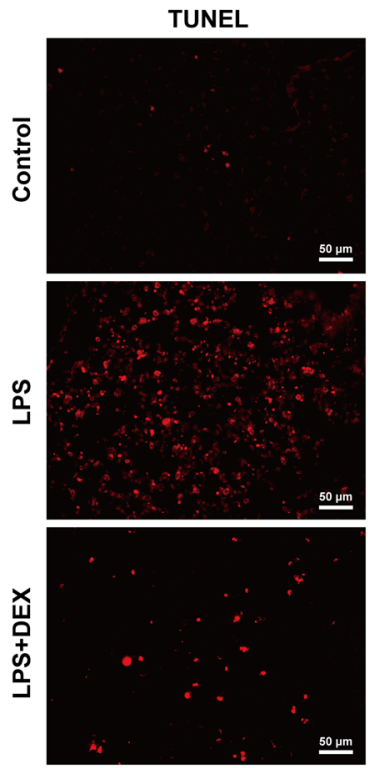

D

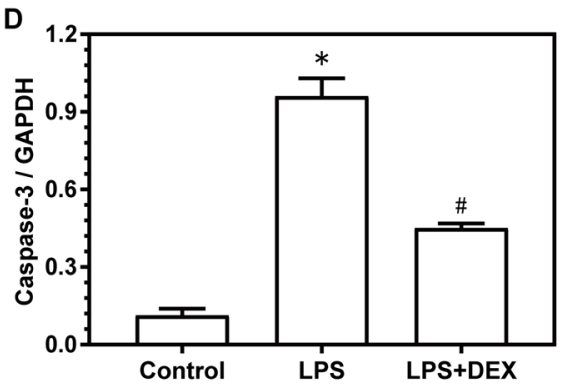

DAPI
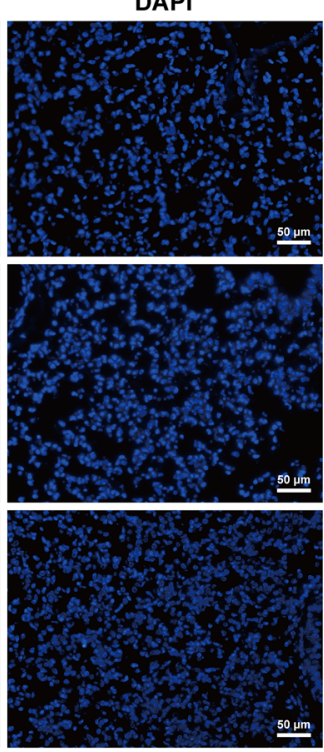
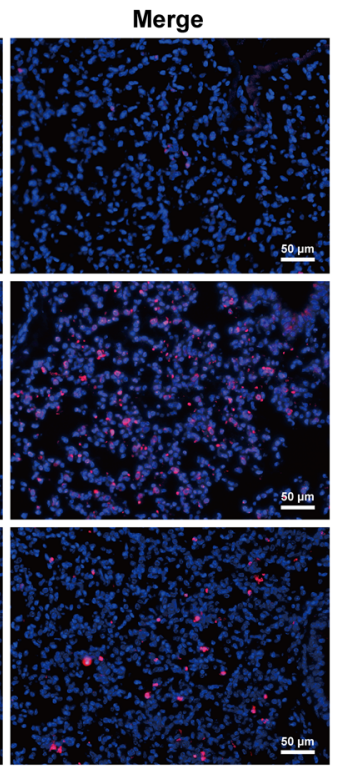

B

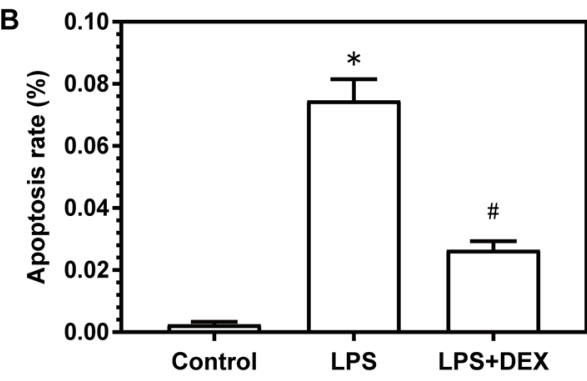

C

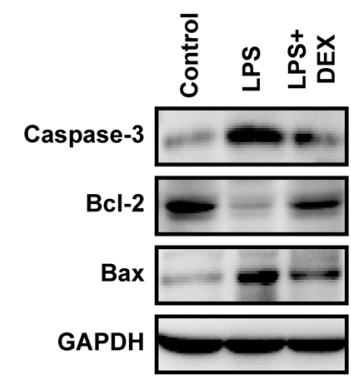

$\mathbf{F}$

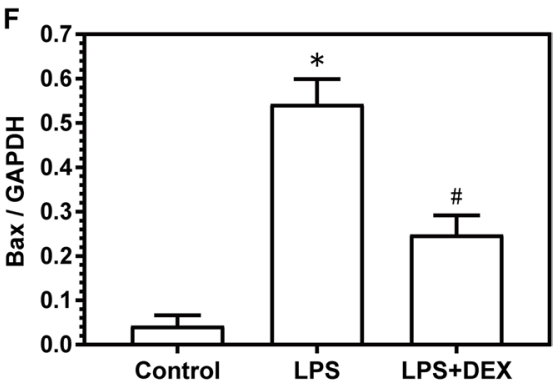

Fig. 4 Dexmedetomidine decreased LPS-induced lung tissue apoptosis in mice. a, b Representative images and semiquantitative analysis of lung tissue apoptosis (TUNEL staining, scale bar $=50 \mu \mathrm{m}$ ). $\mathbf{c}-\mathbf{f}$ Representative Western blot images and quantitative analysis of Cas- pase-3, Bcl-2, and Bax. All of the experiments were repeated at least three times. The results are expressed as mean $\pm \operatorname{SEM}(n=10 /$ group). ${ }^{*} p<0.05$, vs. control group; ${ }^{*} p<0.05$, vs. LPS group 


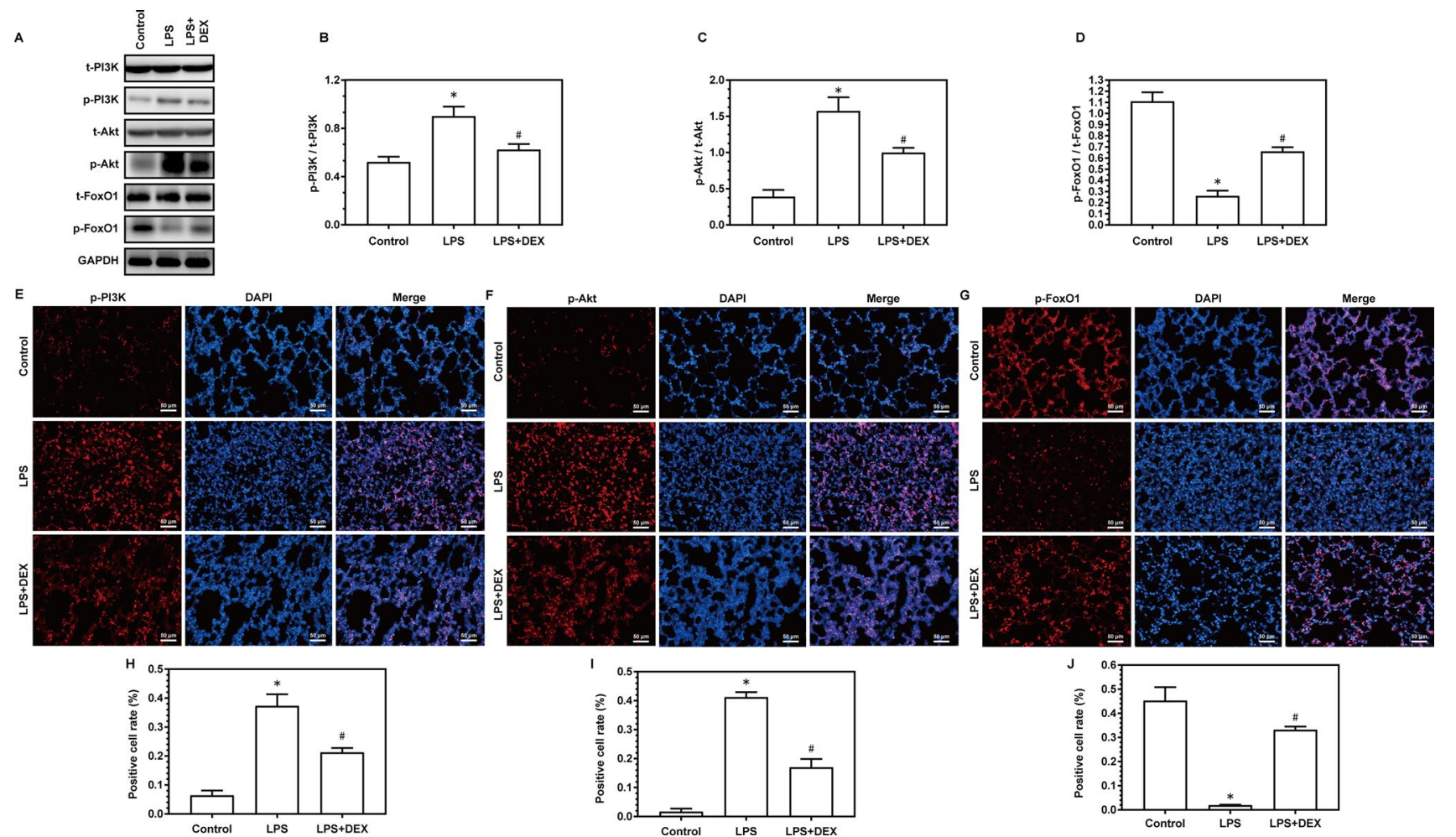

Fig. 5 Dexmedetomidine reversed the effects of LPS on the PI3K/Akt /FoxO1 signaling pathway. a-d Representative images of totalyPI3K (t-PI3K), p-PI3K, total Akt (t-Akt), p-Akt, total FoxO1 (t-FoxO1), and $\mathrm{p}$-FoxO1. e-j Representative immunofluorescent staining images

data indicated that DEX ameliorated LPS-induced acute lung injury partly through the PI3K/Akt/FoxO1 signaling pathway.

\section{BEZ235 pretreatment abolished the effects of DEX on LPS-induced acute lung injury}

LY294002 has an inhibitory effect of PI3K, but IC50 of LY294002 is quite high, so, there always is a possibility of a non-specific effect. Therefore, a PI3K inhibitor (BEZ235) was used to avoid the non-specific effects of LY294002 on LPS-induced lung injury. Compared with the LPS group, BEZ235, a PI3K inhibitor, pretreatment significantly decreased the expression levels of p-PI3K, p-Akt, p-FoxO1, IL-1 $\beta$, IL-6, MDA5, IRE-a, Caspase-3 and Bax, and increased the expression levels of IL-10, SOD-1 and Bcl-2 as compared to the LPS group (Fig. 7, $p<0.05)$. However, BEZ235 pretreatment significantly abolished the inhibitory effects of DEX on LPS-induced lung inflammation, oxidative stress, and apoptosis (Fig. 7, $p<0.05$ ). These data indicated that DEX ameliorated LPSinduced acute lung injury partly through the PI3K/Akt/ FoxO1 signaling pathway. and semiquantitative analysis of p-PI3K, p-Akt, and p-FoxO1. All of the experiments were repeated at least three times. The results are expressed as mean $\pm \mathrm{SEM}$ ( $n=10$ /group). ${ }^{*} p<0.05$, vs. control group; ${ }^{\#} p<0.05$, vs. LPS group

\section{Discussion}

Inflammation and apoptosis have been reported to play key roles in acute lung injury, which is often characterized by leukocyte infiltration, principally neutrophils and macrophages, in the lungs [21, 22]. Accumulating evidence suggests that several pathological changes occur in the lungs after LPS treatment, including inflammation, oxidative stress, and apoptosis, which is a well-known experimental model to assess the effects of drugs on acute lung injury $[23,24]$. The present study found that DEX significantly inhibited LPS-induced increases in the lung weight/body weight ratio and W/D ratio and alleviated inflammatory cell infiltration and the production of proinflammatory factors, oxidative stress, and tissue apoptosis. DEX treatment also decreased p-PI3K and p-Akt expression and increased p-FoxO1 expression. While LY294002 or BEZ235 pretreatment significantly abolished the inhibitory effects of DEX on LPS-induced lung inflammation, oxidative stress, and apoptosis. These data suggest that DEX ameliorates LPS-induced acute lung injury partly through the PI3K/Akt/FoxO1 signaling pathway.

Numerous preliminary results showed that lung resident cells including alveolar macrophages and epithelial cells are 

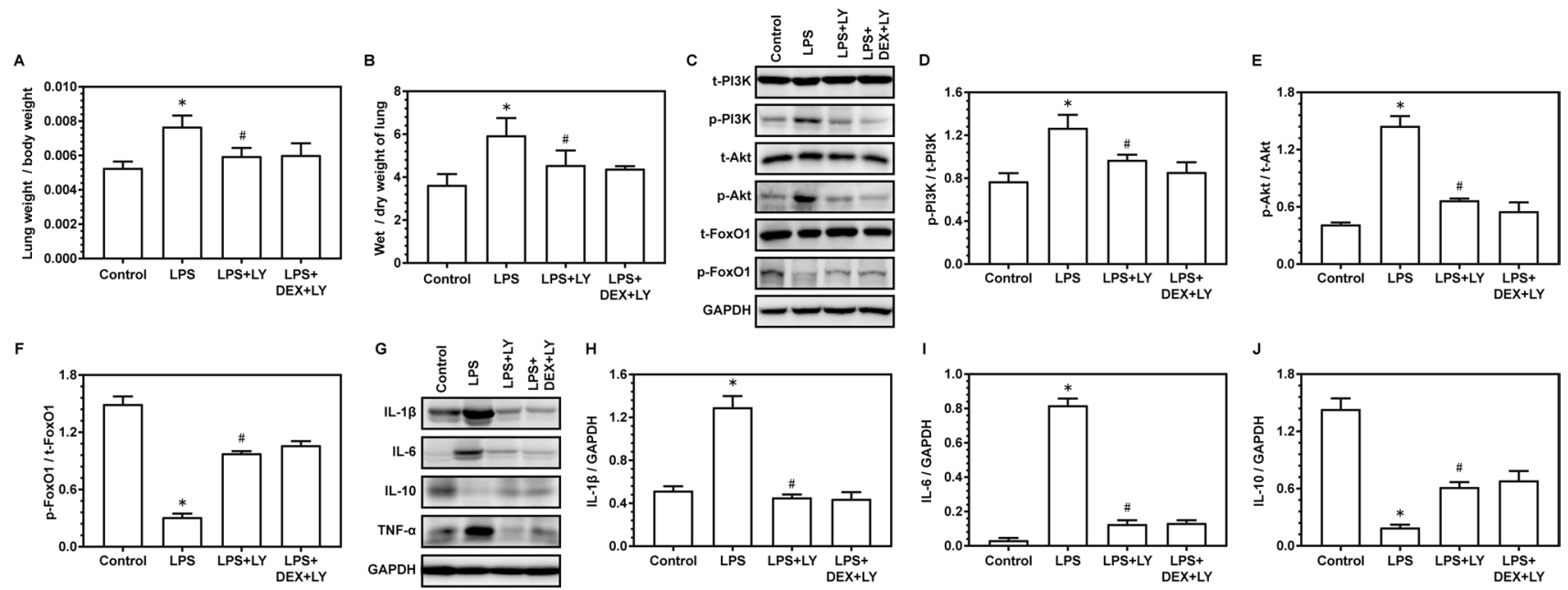

K

L

$\mathbf{M}$

N
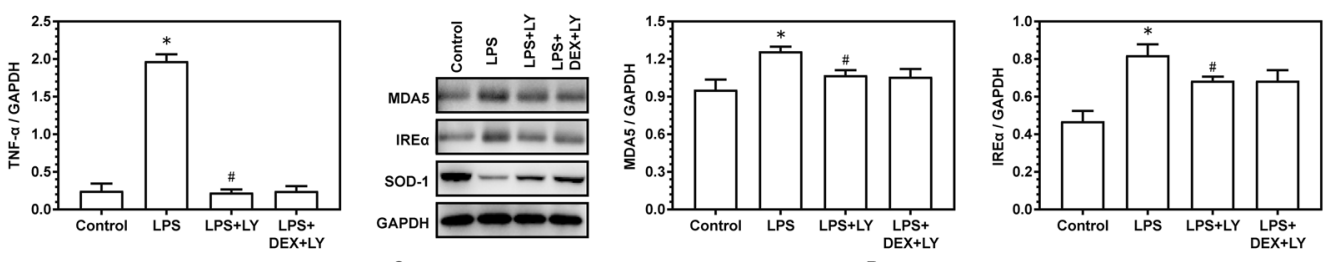

0
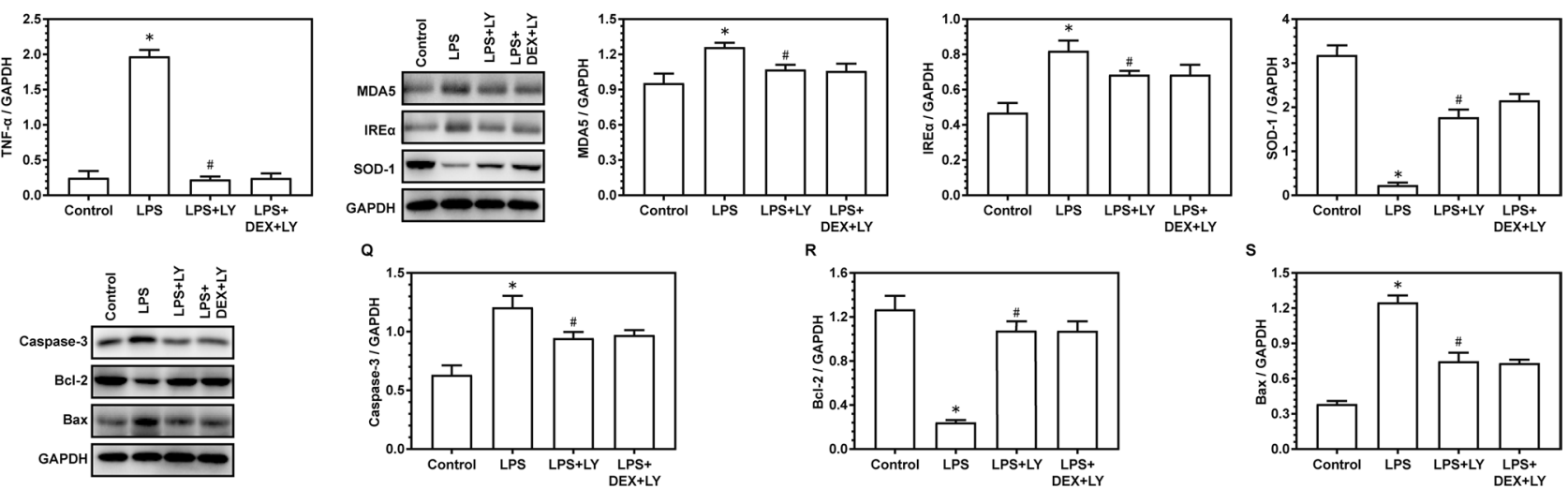

Fig. 6 LY294002 pretreatment abolished the effects of DEX on LPS-induced acute lung injury. a, b Lung weight/body weight ratio and wet/dry ratio. c-f Representative images of total PI3K (t-PI3K), p-PI3K, total Akt (t-Akt), p-Akt, total FoxO1 (t-FoxO1), and p-FoxO1. g-k Representative images and quantitative analysis of IL-1 $\beta$, IL-6, IL-10, and TNF- $\alpha$. l-o Representative images and

activated in the early phase of LPS treatment, which induced up-regulation of pro-inflammatory cytokines including IL-6 and TNF-a [25, 26]. In the present study, DEX dramatically inhibited LPS-induced inflammatory cell infiltration in the lungs and the production of proinflammatory factors, oxidative stress, and tissue apoptosis. Similarly, Liu et al. [15] reported that DEX pretreatment decreased LPS-induced lung injury scores, decreased myeloperoxidase activity, and decreased inflammatory cell infiltration in the lungs. DEX pretreatment also decreased the expression of Toll-like receptor 4 (TLR-4) and NF- $\mathrm{KB}$ in the lungs, suggesting that it protects against LPS-induced acute lung injury by suppressing activation of the TLR-4/NF- $\mathrm{B}$ signaling pathway. Meanwhile, DEX pretreatment was previously reported to attenuate lung injury and reduce the production of inflammatory factors by decreasing the levels of NACHT, LRR, and PYD domains containing protein 3 (NLRP3) and autocleavage of caspase-1 [16]. Moreover, DEX was also showed to

quantitative analysis of MDA5, IRE $\alpha$, and SOD-1. p-s Representative images and quantitative analysis of Caspase-3, Bcl-2, and Bax. All of the experiments were repeated at least three times. The results are expressed as mean $\pm \operatorname{SEM}\left(n=10\right.$ /group). ${ }^{*} p<0.05$, vs. control group; ${ }^{\#} p<0.05$, vs. LPS group

play a crucial role in LPS-induced acute lung injury, possible via the high mobility group box 1-mediated TLR-4/NF- $\mathrm{KB}$ and $\mathrm{PI} 3 \mathrm{~K} / \mathrm{Akt} / \mathrm{mammalian} / \mathrm{mechanistic}$ target of rapamycin pathways [27]. In addition to its antiinflammatory properties, DEX pretreatment has been reported to reduce LPS-induced human lung fibroblast apoptosis by inhibiting gap junction proteins and reducing the protein expression of connexin-43 [28]. Apoptosis is essential for the selective elimination of cells, and it plays an important role in the occurrence and development of acute lung injury. The inhibition of lung cells apoptosis might increase the animal survival rate in LPS-induced acute lung injury [29]. DEX pretreatment ameliorated LPS-induced lung inflammation and oxidative stress by activating the Nrf2/Keap1 signaling pathway and regulating the expression of antioxidant genes [30]. These data suggest that DEX effectively alleviates LPS-induced lung inflammation, oxidative stress, and apoptosis. 

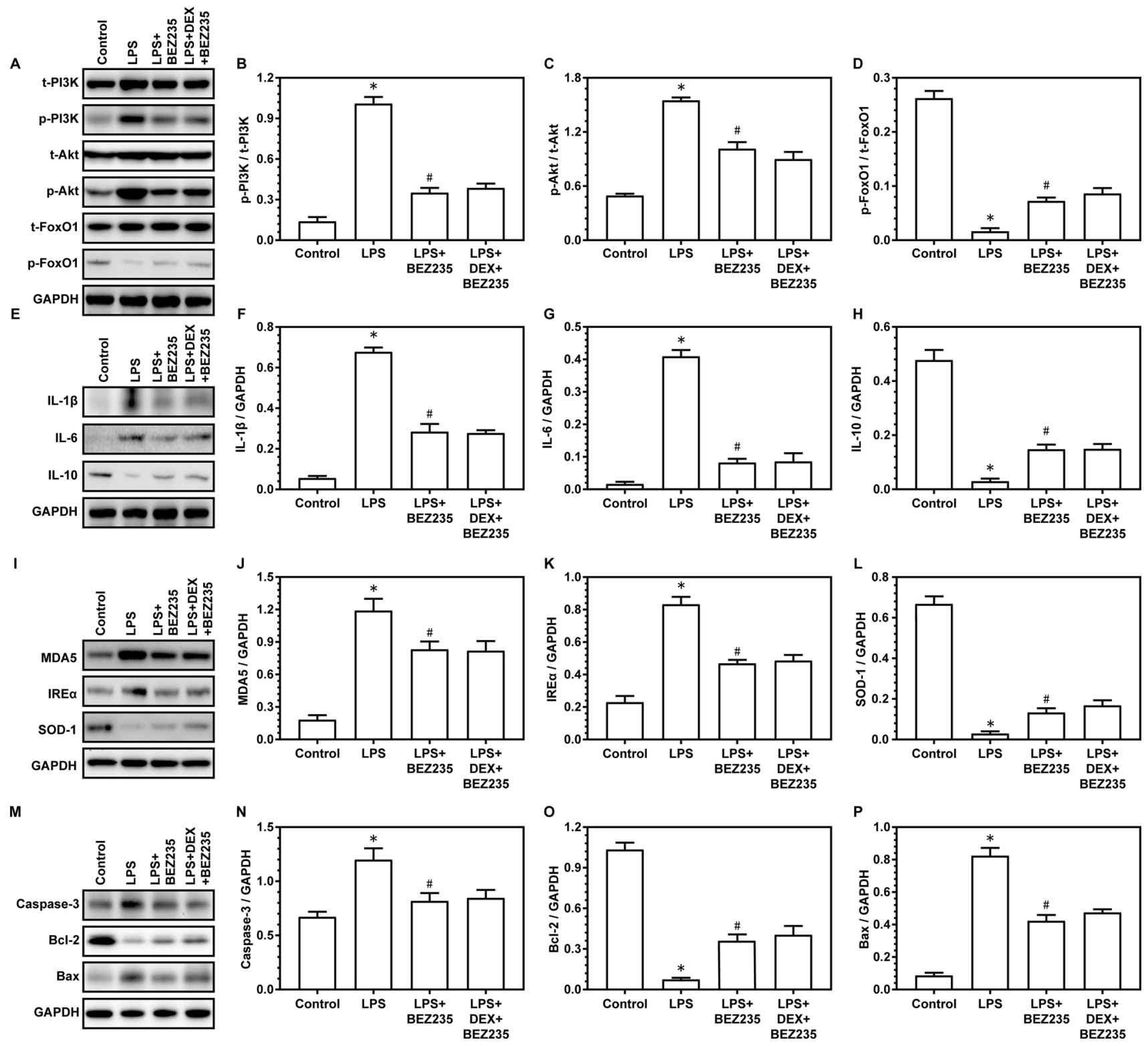

Fig. 7 BEZ235 pretreatment abolished the effects of DEX on LPSinduced acute lung injury. a-d Representative images and quantitative analysis of total PI3K (t-PI3K), p-PI3K, total Akt (t-Akt), p-Akt, total FoxO1 (t-FoxO1), and p-FoxO1. e-h Representative images and quantitative analysis of IL-1 $\beta$, IL- 6 and IL-10. i-l Representative

images and quantitative analysis of MDA5, IRE $\alpha$, and SOD-1. m-p Representative images and quantitative analysis of Caspase-3, Bcl-2, and Bax. All of the experiments were repeated at least three times. The results are expressed as mean \pm SEM ( $n=10$ /group). ${ }^{*} p<0.05$, vs. control group; $\# p<0.05$, vs. LPS group

It has been reported that activation of PI3K/Akt signaling pathway protects tissues or cells against LPS- and CLPinduced-acute lung injury through inhibiting cell apoptosis $[31,32]$. To further investigate whether DEX protects against LPS-induced lung injury through the PI3K/Akt/FoxO1 signaling pathway, we pretreated the samples with the PI3K/Akt inhibitor LY294002 and BEZ235 before the induction of LPS-induced lung injury. A study was reported by Antonia Patruno et al. that pre-administration of LY294002 (a Akt inhibitor) and PD980559 (a ERK inhibitor) significantly inhibited Nrf2 nuclear translocation and $\mathrm{HO}-1$ protein expression induced by LPS [33]. Moreover, administration of artesunate $(15 \mathrm{mg} / \mathrm{kg})$ was found to significantly attenuate the levels of IL-6, TNF- $\alpha$, caspase 3, MDA, SOD and GSH-Px as compared to the LPS group at $24 \mathrm{~h}$, and this attenuation was reversed by LY294002 [34]. In the present study, our data demonstrated that DEX significantly ameliorated the LPS-induced activation of PI3K/Akt protein and increased FoxO1 phosphorylation. LY294002 pretreatment abolished the protective effects of DEX on LPS-induced 
inflammatory cell infiltration, oxidative stress, and apoptosis in the lungs. Similar to our results, a previous study found that DEX significantly reduced lung injury in rats with obstructive jaundice through activation of the PI3K/ Akt/HIF-1 $\alpha$ signaling pathway [35]. DEX decreased the expression of IL-6 and TNF- $\alpha$ in obstructive jaundice rats [36]. DEX was also shown to protect against ischemia/reperfusion-induced lung injury through activation of the PI3K/ Akt/HIF-1 $\alpha$ signaling pathway [37]. Similar results were reported by $\mathrm{Li}$ et al. [38] who found that DEX alleviated renal ischemia/reperfusion-induced lung apoptosis, and significantly increased p-Akt expression in the lungs, and these effects were abolished by LY294002, suggesting that DEX attenuates lung apoptosis that is induced by ischemia/reperfusion injury possibly through the $\alpha 2$-adrenergic receptor $(\alpha 2 \mathrm{AR}) / \mathrm{PI} 3 \mathrm{~K} / \mathrm{Akt}$ pathway. Additionally, Li et al. [39] found that DEX dramatically attenuated post-cardiopulmonary bypass surgery-induced lung injury partially through activation of the PI3K/Akt signaling pathway and the inhibition of lung tissue apoptosis. More interestingly, our study showed that BEZ235 pretreatment was also revealed to abolish the inhibitory effects of DEX on LPS-induced lung inflammation, oxidative stress, and apoptosis. Consistent with these findings, the present data indicated that LY294002 pretreatment markedly abolished the protective effects of DEX on LPS-induced lung inflammation, oxidative stress, and apoptosis in mice. Several studies demonstrated that pretreatment with DEX ameliorated LPS-induced acute lung injury [40-42], and DEX was also reported to alleviate lung ischemia-reperfusion-induced acute lung injury [43], and to alleviate lung ventilation (OLV) during video-assisted thoracoscopic surgery (VATS) lead to acute lung injury partly through activating PI3K/Akt signaling pathway [44]. However, unknown are whether DEX ameliorates LPSinduced lung inflammation, oxidative stress, and apoptosis and whether the protective effect of DEX involves the PI3K/ Akt/FoxO1 signaling pathway. Therefore, the novelty of this study is to explore for the first time that DEX alleviates LPSinduced lung injury by targeting PI3K/Akt/FoxO1 signaling pathway. However, the limitation of this study is that the dose of DEX is quite high and may have an effect on the whole body. Moreover, DEX is usually given by continuous infusion in the clinic, which is different from that of intraperitoneal injection in this study.

In summary, the present study suggests that DEX protects against LPS-induced acute lung injury, including lung inflammation, oxidative stress, and apoptosis, and these protective effects at least partially occurred through the $\mathrm{PI} 3 \mathrm{~K} / \mathrm{Akt} / \mathrm{FoxO} 1$ signaling pathway.

Acknowledgements We thank Traci Raley, MS, ELS, from Liwen Bianji, Edanz Editing China, for editing a draft of this manuscript.

\section{Compliance with ethical standards}

Conflict of interests The authors declare that there are no competing interests.

\section{References}

1. Li X, Yuan Z, Chen J, Wang T, Wen F. Microarray analysis reveals the changes of circular RNA expression and molecular mechanism in acute lung injury mouse model. J Cell Biochem. 2019;120:16658.

2. Kumari A, Dash D, Singh R. Lipopolysaccharide (LPS) exposure differently affects allergic asthma exacerbations and its amelioration by intranasal curcumin in mice. Cytokine. 2015;76(2):334-42.

3. Wang D, Wang X, Tong W, Cui Y, Li X, Sun H. Umbelliferone alleviates lipopolysaccharide-induced inflammatory responses in acute lung injury by down-regulating TLR4/MyD88/NF-кB signaling. Inflammation. 2019;42:440.

4. Mutt SJ, Raza GS, Makinen MJ, Keinanen-Kiukaanniemi S, Jarvelin MR and Herzig KH: Vitamin D Deficiency Induces Insulin Resistance and Re-supplementation Attenuates Hepatic Glucose Output Via the PI3K-AKT-FOXO1 Mediated Pathway. Mol Nutr Food Res. 2019; e1900728.

5. Wu Z, Niu T, Xiao W. Uev1A promotes breast cancer cell survival and chemoresistance through the AKT-FOXO1-BIM pathway. Cancer Cell Int. 2019;19:331.

6. Dang LTH, Aburatani T, Marsh GA, Johnson BG, Alimperti S, Yoon CJ, Huang A, Szak S,Nakagawa N, Gomez I, Ren S, Read SK, Sparages C, Aplin AC, Nicosia RF, Chen C, Ligresti G, Duffield JS. Hyperactive FOXO1 results in lack of tip stalk identity and deficient microvascular regeneration during kidney injury. Biomaterials. 2017;141:314-29.

7. Li L, Li X, Zhang Z, Liu L, Liu T, Li S, Liu S, Zhou Y, Liu F. Effects of hydrogen-rich water on the PI3K/AKT signaling pathway in rats with myocardial ischemia-reperfusion injury. Curr Mol Med. 2019.

8. Pitaloka DMI, Ko CH, Lin MT, Yeh SL, Yeh CL. Glutamine administration promotes hepatic glucose homeostasis through regulating the PI3K/Akt pathway in high-fat diet-induced obese mice with limb ischemia. Nutrition. 2019;68:45-53.

9. Shi J, Yu J. PI3K/Akt pathway-mediated HO-1 induction regulates mitochondrial quality control and attenuates endotoxin-induced acute lung injury. Lab Invest. 2019;99:1795-809.

10. Sun K, Huang R, Yan L, Li D-T, Liu Y-Y, Wei X-H, Cui Y-C, Pan C-S, Fan J-Y, Wang X, Han J-Y. Schisandrin attenuates lipopolysaccharide-induced lung injury by regulating TLR-4 and Akt/ FoxO1 signaling pathways. Front Physiol. 2018;9:1104.

11. Elbakry A-E, Sultan W-E, Ibrahim E. A comparison between inhalational (Desflurane) and total intravenous anaesthesia (Propofol and dexmedetomidine) in improving postoperative recovery for morbidly obese patients undergoing laparoscopic sleeve gastrectomy: A double-blinded randomised controlled trial. J Clin Anesth. 2018;45:6.

12. Feng X, Guan W, Zhao Y, Wang C, Song M, Yao Y, Yang T, Fan H. Dexmedetomidine ameliorates lipopolysaccharide-induced acute kidney injury in rats by inhibiting inflammation and oxidative stress via the GSK-3 $\beta /$ Nrf2 signaling pathway. J Cell Physiol. 2019;234:18994.

13. Xu Y, Zhang R, Li C, Yin X, Lv C, Wang Y, Zhao W, Zhang $\mathrm{X}$. Dexmedetomidine attenuates acute lung injury induced by lipopolysaccharide in mouse through inhibition of MAPK pathway. Fundam Clin Pharmacol. 2015;29:462-71. 
14. Song Q, Lin L, Chen L, Cheng L, Zhong W. Co-administration of $\mathrm{N}$-acetylcysteine and dexmedetomidine plays a synergistic effect on protection of LPS-induced acute lung injury via correcting Th1/Th2/Th17 cytokines imbalance. Clin Exp Pharmacol Physiol. 2019;47(2):294-301.

15. Liu J, Huang X, Hu S, He H, Meng Z. Dexmedetomidine attenuates lipopolysaccharide induced acute lung injury in rats by inhibition of caveolin-1 downstream signaling. Clin Exp Pharmacol Physiol. 2019;118:109314.

16. Zhang Y, Wang X, Liu Z, Yu L. Dexmedetomidine attenuates lipopolysaccharide induced acute lung injury by targeting NLRP3 via miR-381. J Biochem Mol Toxicol. 2018;32:e22211.

17. Wu Y, Liu Y, Huang H, et al. Dexmedetomidine inhibits inflammatory reaction in lung tissues of septic rats by suppressing TLR4/NF-кB pathway. Mediators Inflamm 2013: 1-9.

18. Samary CS, Moraes L, Santos CL, Huhle R, Santos RS, Ornellas DS, Felix NS, Capelozzi VL, Schanaider A, Pelosi P, de Abreu MG, Rocco PRM, Silva PL. Lung functional and biologic responses to variable ventilation in experimental pulmonary and extrapulmonary acute respiratory distress syndrome. Crit Care Med. 2016;44:1.

19. Li L-F, Liao S-K, Lee C-H, Huang C-C, Quinn DA. Involvement of Akt and endothelial nitric oxide synthase in ventilation-induced neutrophil infiltration: a prospective, controlled animal experiment. Crit Care. 2007;11:R89.

20. Fròsina G, Profumo A, Marubbi D, Marcello D, Ravetti JL, Daga A. ATR kinase inhibitors NVP-BEZ235 and AZD6738 effectively penetrate the brain after systemic administration. Radiat Oncol. 2018;13:76.

21. Wang P, Hou Y, Zhang W, Zhang H, Che X, Gao Y, Liu Y, Yang D, Wang J, Xiang R,Zhao M, Yang J. Pseudoginsenoside-F11 attenuates lipopolysaccharide-induced acute lung injury by suppressing neutrophil infiltration and accelerating neutrophil clearance. Inflammation. 2019;42(5):1857-68.

22. Jiang Q, Wang S, Shi Y. Posttreatment With LYRM03 protects rats from acute lung inflammation induced by lipopolysaccharide via suppressing the NF-kappaB/MyD88/TLR4 Axis. Inflammation. 2019;243:316-24.

23. Su VY, Lin CS, Hung SC, Yang KY. Mesenchymal stem cellconditioned medium induces neutrophil apoptosis associated with inhibition of the NF-kappaB pathway in endotoxin-induced acute lung injury. Int J Mol Sci. 2019;20:2208.

24. Kosutova P, Mikolka P, Kolomaznik M, et al. Reduction of lung inflammation, oxidative stress and apoptosis by the PDE4 inhibitor roflumilast in experimental model of acute lung injury. Physiol Res. 2018;67:S645-s654.

25. Lee B-W, Ha J-H, Shin H-G, Jeong S-H, Jeon D-B, Kim J-H, Park J-Y, Kwon H-J, Jung K, Lee W-S, Kim H-Y, Kim S-H, Jang H-J, Ryu Y-B, Lee I-C. Spiraea prunifolia var. attenuates oxidative stress and inflammatory responses in a murine model of lipopolysaccharide-induced acute lung injury and TNF- $\alpha$-stimulated NCIH292 cells. Antioxidants (Basel). 2020;9:198.

26. Jiang Z, Chen Z, Hu L, Qiu L, Zhu L. Calreticulin blockade attenuates murine acute lung injury by inducing polarization of M2 subtype macrophages. Front Immunol. 2020;11:11.

27. Meng L, Li L, Lu S, Li K, Su Z, Wang Y, Fan X, Li X, Zhao G. The protective effect of dexmedetomidine on LPS-induced acute lung injury through the HMGB1-mediated TLR4/NF- $\mathrm{KB}$ and PI3K/Akt/mTOR pathways. Mol Immunol. 2017;94:7-17.

28. Zhang Y, Tan X, Xue L. The alpha2-adrenoreceptor agonist dexmedetomidine protects against lipopolysaccharide-induced apoptosis via inhibition of gap junctions in lung fibroblasts. Biochem Biophys Res Commun. 2018;495:92.

29. Liu L, Liu F, Sun Z, Peng Z, You T, Yu Z. LncRNA NEAT1 promotes apoptosis and inflammation in LPS-induced sepsis models by targeting miR-590-3p. Exp Ther Med. 2020;20:3290-300.
30. Yan X, Cheng X, Zhou L, He X, Chen H. Dexmedetomidine alleviates lipopolysaccharide-induced lung injury in Wistar rats. Oncotarget. 2017;8:44410.

31. Li R, Zou X, Huang H, Yu Y, Zhang H, Liu P, Pan S, Ouyang Y, Shang Y. HMGB1/PI3K/Akt/mTOR signaling participates in the pathological process of acute lung injury by regulating the maturation and function of dendritic cells. Front Immunol. 2020;11:1104.

32. Huang C-Y, Deng J-S, Huang W-C, Jiang W-P, Huang G-J. Attenuation of lipopolysaccharide-induced acute lung injury by hispolon in mice, through regulating the TLR4/PI3K/Akt/mTOR and Keap1/Nrf2/HO-1 pathways, and suppressing oxidative stressmediated ER stress-induced apoptosis and autophagy. Nutrients. 2020;12:1742.

33. Patruno A, Costantini E, Ferrone A, Pesce M, Diomede F, Trubiani O, Reale M. Short ELF-EMF exposure targets SIRT1/Nrf2/ HO-1 signaling in THP-1 cells. Int J Mol Sci. 2020;21:7284.

34. Zhang E, Wang J, Chen Q, Wang Z, Li D, Jiang N, Ju X. Artesunate ameliorates sepsis-induced acute lung injury by activating the mTOR/AKT/PI3K axis. Gene. 2020;759:144969.

35. Xie Y, Guo C, Liu Y, Shi L, Yu J. Dexmedetomidine activates the PI3K/Akt pathway to inhibit hepatocyte apoptosis in rats with obstructive jaundice. Exp Ther Med. 2019;18:4461-6.

36. Shi L, Guo C, Xie Y, Liu Y, Wu F. Dexmedetomidine attenuates lung injury in obstructive jaundice rats through PI3K/Akt/HIF1alpha signaling pathway. Arch Med Res. 2019;50:233-40.

37. Zhang W, Zhang J-Q, Meng F-M, Xue F-S. Dexmedetomidine protects against lung ischemia-reperfusion injury by the PI3K/ Akt/HIF-1 $\alpha$ signaling pathway. J Anesth. 2016;30:826-33.

38. Li J, Chen Q, He X, Alam A, Ning J, Yi B, Lu K, Gu J. Dexmedetomidine attenuates lung apoptosis induced by renal ischemiareperfusion injury through $\alpha 2 \mathrm{AR} / \mathrm{PI} 3 \mathrm{~K} / \mathrm{Akt}$ pathway. J Transl Med. 2018;16:78.

39. Li J, Dou X, Li D, He M, Han M, Zhang H. Dexmedetomidine ameliorates post-CPB lung injury in rats by activating the PI3K/ Akt pathway. J Invest Surg. 2019; 1-8.

40. Ding D, Xu S, Zhang H, Zhao W, Zhang X, Jiang Y, Wang P, Dai Z, Zhang J. 3-Methyladenine and dexmedetomidine reverse lipopolysaccharide-induced acute lung injury through the inhibition of inflammation and autophagy. Exp Ther Med. 2018;15:3516-22.

41. Fu C, Dai X, Yang Y, Lin M, Cai Y, Cai S. Dexmedetomidine attenuates lipopolysaccharide-induced acute lung injury by inhibiting oxidative stress, mitochondrial dysfunction and apoptosis in rats. Mol Med Rep. 2017;15:131-8.

42. Sun Y, Xia Y, Liu X, Liu J, He W, Ye H, Yuan X. Dexmedetomidine alleviates LPS-induced acute lung injury via regulation of the p38/HO-1 pathway. Mol Med Rep. 2020;22:2442-50.

43. Liang S, Wang Y, Liu Y. Dexmedetomidine alleviates lung ischemia-reperfusion injury in rats by activating PI3K/Akt pathway. Eur Rev Med Pharmacol Sci. 2019;23:370-7.

44. Zhu L, Zhang Y, Zhang Z, Ding X, Gong C, Qian Y. Activation of PI3K/Akt/HIF- $1 \alpha$ signaling is involved in lung protection of dexmedetomidine in patients undergoing video-assisted thoracoscopic surgery: a pilot study. Drug Design Dev Therapy. 2020;14:5155-66.

Publisher's Note Springer Nature remains neutral with regard to jurisdictional claims in published maps and institutional affiliations. 